\title{
Correction to: Rosacea
}

\author{
Shlomit Halachmi
}

\section{Correction to: Chapter 58 in: M. Alam (ed.), Evidence-Based Procedural Dermatology, https://doi.org/10.1007/978-3-030-02023-1_58}

In Chapter 58, the affiliation of the chapter author was incorrect.

The affiliation is now corrected and updated. 